ARTICLE

DOI: $10.1038 / \mathrm{s} 41467-018-04502-3$

\title{
Robust nonfullerene solar cells approaching unity external quantum efficiency enabled by suppression of geminate recombination
}

\author{
Derya Baran (10 1, Nicola Gasparini (10) 1,2, Andrew Wadsworth (10 3, Ching Hong Tan ${ }^{3}$, Nimer Wehbe ${ }^{1,4}$,
} Xin Song (1) 1, Zeinab Hamid ${ }^{3}$, Weimin Zhang ${ }^{1}$, Marios Neophytou1, Thomas Kirchartz ${ }^{1,6}$, Christoph J. Brabec ${ }^{2,7}$, James R. Durrant ${ }^{3,8}$ \& lain McCulloch ${ }^{1,3}$

Nonfullerene solar cells have increased their efficiencies up to 13\%, yet quantum efficiencies are still limited to $80 \%$. Here we report efficient nonfullerene solar cells with quantum efficiencies approaching unity. This is achieved with overlapping absorption bands of donor and acceptor that increases the photon absorption strength in the range from about 570 to $700 \mathrm{~nm}$, thus, almost all incident photons are absorbed in the active layer. The charges generated are found to dissociate with negligible geminate recombination losses resulting in a short-circuit current density of $20 \mathrm{~mA} \mathrm{~cm}^{-2}$ along with open-circuit voltages $>1 \mathrm{~V}$, which is remarkable for a $1.6 \mathrm{eV}$ bandgap system. Most importantly, the unique nano-morphology of the donor:acceptor blend results in a substantially improved stability under illumination. Understanding the efficient charge separation in nonfullerene acceptors can pave the way to robust and recombination-free organic solar cells.

\footnotetext{
${ }^{1}$ King Abdullah University of Science and Technology (KAUST), KAUST Solar Center (KSC), Physical Sciences and Engineering Division (PSE), Thuwal 23955-6900, Saudi Arabia. ${ }^{2}$ Institute of Materials for Electronics and Energy Technology (I-MEET), Friedrich-Alexander-University Erlangen-Nuremberg, Erlangen, Germany. ${ }^{3}$ Department of Chemistry and Centre for Plastic Electronics, Imperial College London, London SW7 2AZ, UK. ${ }^{4}$ King Abdullah University of Science and Technology (KAUST), Core Labs, Thuwal 23955-6900, Saudi Arabia. ${ }^{5}$ IEK5-Photovoltaics, Forschungszentrum Jülich, 52425 Jülich, Germany. ${ }^{6}$ Faculty of Engineering and CENIDE, University of Duisburg-Essen, Carl-Benz-Straße 199, 47057 Duisburg, Germany. ${ }^{7}$ ZAE Bayern, Immerwahrstraße 2, Erlangen 91058, Germany. ${ }^{8}$ SPECIFIC IKC, Swansea University, Baglan Bay Innovation Centre, Port Talbot, Swansea SA12 7AX, UK. Correspondence and requests for materials should be addressed to D.B. (email: derya.baran@kaust.edu.sa) or to N.G. (email: nicola.gasparini@kaust.edu.sa)
} 
S olution-processed organic solar cells have traditionally relied mostly on the strong photon absorption provided by molecularly designed small molecule and polymer donor materials ${ }^{1}$. In order to dissociate photo-generated excitons created on the donor molecules, an electron accepting molecule is required. Traditionally, high power conversion efficiencies (PCEs) that are coupled with notable external quantum efficiencies (EQEs) had only been obtained using fullerene-based acceptor molecules. While fullerene acceptors allow ultrafast charge separation and fairly efficient charge transport ${ }^{2}$, their optical absorption is poor in comparison to many polymers and other small molecules in the visible range. Therefore, there have always been efforts to replace fullerene acceptors with molecules, which combine the advantages of fullerenes with a higher absorption strength $^{3}$. Only recently, efficient nonfullerene acceptor molecules (NFAs) have emerged that compete or even outperform fullerenes when mixed with high-efficiency donor molecules ${ }^{4-8}$. In addition to the strong absorption of light, the success of NFAs can be in part attributed to the tunability of their optical properties ${ }^{9}$. By attaching strongly electron-withdrawing groups to an electrondonating core, it is possible to achieve a reasonably narrow bandgap due to the push-pull effect ${ }^{3,10-12}$ while maintaining the planarity of the molecule. This will result in a large overlap between the highest occupied molecular orbital and lowest unoccupied molecular orbital (LUMO) of the acceptor ${ }^{13-15}$. This orbital overlap leads to a greater oscillator strength and therefore a stronger absorption for light. In addition to the substantially improved absorption, polymer:NFA solar cells reported to date have frequently exhibited impressive open-circuit voltages when compared to their absorption onset ${ }^{16}$. There has been a recent report showing exciton separation in the picosecond range enabling efficient solar cells up to $9.5 \%{ }^{17}$. However, such polymer:NFA devices have typically shown relatively modest EQEs, attributed to geminate recombination losses most likely associated with the small energetic offset driving charge separation in these devices ${ }^{16,18}$. Thus, the ability to reduce these losses while maintaining a rather high voltage for EQEs $>80 \%$ has therefore become imperative to improve efficiency in polymer:NFA systems.

PBDTTT-EFT, more commonly known as PCE10 (or PTB7Th), has been used extensively with NFAs and to date efficiencies $(\eta)$ up to $11 \%$ have been achieved ${ }^{7,19}$. Early examples of PBDTTT-EFT:NFA systems in organic solar cells were in allpolymer blends. Initially, these blends were only able to achieve modest efficiencies (3-5\%) due to the sub-optimal morphologies resulting from the unfavorable mixing of two polymer components in a blend, resulting in large recombination losses, which was accompanied by low EQEs with a maxima around $50 \%{ }^{20,21}$. The PCE of all-polymer systems were later improved by addressing these morphological issues, creating more intermixed bulk heterojunctions possessing more balanced charge-carrier mobilities in the blends, thereby suppressing recombination. As a result, these all-polymer blends were able to achieve an EQE maximum of $65-70 \% 22-24$. The development of small-molecule NFAs allowed further improvements in the PBDTTT-EFT:NFA systems due to the lack of entropic driving force of polymers for mixing with small molecules ${ }^{7}$. The improved morphologies due to favorable mixing and higher absorption coefficients were reflected in improved PCEs in devices using small-molecule NFAs and coincided with greater EQEs in devices $(80 \%)^{25-27}$. Despite these advances, the maximum potential of PBDTTT-EFT solar cells have not been reached with $V_{\text {oc }}$ values $>1 \mathrm{~V}$ and EQEs $>80 \%$ thus far.

In this work, we report PBDTTT-EFT devices with an indacenodithiophene derivative NFA (EHIDTBR) that are able to achieve efficiencies of $12 \%$, with a $V_{\mathrm{oc}}>1 \mathrm{~V}$ and EQE values around $90 \%$. In the cells with the highest photocurrent, we achieve $J_{\mathrm{sc}}$ of $20 \mathrm{~mA} \mathrm{~cm}^{-2}\left(J_{\mathrm{sc}}\right.$ of $18.5 \mathrm{~mA} \mathrm{~cm}^{-2}$ in the cells with the highest efficiencies), which is exceptionally high for organic solar cells with an absorption onset around $1.6 \mathrm{eV}$. We elucidate the origin of this high $J_{\mathrm{sc}}$ and $\mathrm{EQE}$ with the findings that fast and efficient exciton separation smaller than $10 \mathrm{ps}$ and $>90 \%$ photoluminescence quenching yield followed by an efficient polaron formation with minimal geminate recombination losses. Moreover, depth profile analyses of the active layer of solar cell devices reveal that the strongly miscible character of PBDTTT-EFT and EHIDTBR leads to the formation of a robust microstructure and stable devices under illumination. In contrast, in the case of the fullerene devices, phase separation and demixing were evident over time causing reduced fill factor values even after $24 \mathrm{~h}$. This study directly shows the suppression of geminate recombination for an NFA-based system combined with near unity quantum efficiency, exciton dissociation, and a robust morphology without vertical phase separation, yielding highly efficient $(12 \%)$ and stable organic photovoltaic cells suitable for scale-up and commercial application.

\section{Results}

Photovoltaic characterization. The photovoltaic properties of the solar cells are investigated by constructing an indium tin oxide (ITO)/electron-transport layer (ETL)/active layer ( $d$ of $80-120$ $\mathrm{nm}) / \mathrm{MoO}_{3} / \mathrm{Ag}$ architecture using different ETL modifications based on sol-gel $\mathrm{ZnO}$ and/or polyethylenimine (PEIE) for device optimization. Chemical structures of donor and acceptor molecules and current-voltage $(J-V)$ characteristics of the PBDTTTEFT:EHIDTBR ( $24 \mathrm{mg} \mathrm{ml}^{-1}$ in chlorobenzene solution) devices made using different ETLs in comparison to PBDTTT-EFT: $\mathrm{PC}_{71} \mathrm{BM}$ with $3 \%$ 1,8-diiodooctane (DIO) devices are shown in Fig. $1 \mathrm{a}, \mathrm{b}$, respectively. The key photovoltaic parameters for different ETL modifications in PBDTTT-EFT:EHIDTBR devices using $\mathrm{ZnO}$, solvent modified $\mathrm{ZnO}$ (with $1 \%$ ethanolamine), and PEIE are illustrated in Table 1 . In order to evaluate the performance of EHIDTBR in comparison to fullerenes, PBDTTT-EFT: $\mathrm{PC}_{71} \mathrm{BM}$ devices are constructed following previous reports with DIO $(3 \%)^{28,29}$. The substantially higher $V_{\text {oc }}$ of the nonfullerene devices results from tailored energy-level alignment between the frontier molecular orbitals at the donor/acceptor interface of the PBDTTT-EFT:EHIDTBR blend. The photovoltaic devices from PBDTTT-EFT:EHIDTBR blend exhibit an open-circuit voltage $\left(V_{\mathrm{oc}}\right)$ of $1.03 \mathrm{~V}$ owing to the higher electron affinity (EA) (Supplementary Fig. 1) values of EHIDTBR $(3.90 \mathrm{eV})$ compared to $\mathrm{PC}_{71} \mathrm{BM}(4.1 \mathrm{eV})^{7,30}$, where $V_{\mathrm{oc}}$ was limited to $0.78 \mathrm{~V}$. In addition, a substantial improvement in the short-circuit current $\left(J_{\text {sc }}\right)$ up to $20 \mathrm{mAcm}^{-2}$, an EQE maximum of $90 \%$ and fill factor (FF) of 0.63 values achieved yielding a PCE of $12 \%$ for PBDTTT-EFT: EHIDTBR devices without any thermal or solvent treatments. There has been a study about nonfullerene acceptor with PCEs up to $11 \%{ }^{19}$, by far the highest efficiency reported using PBDTTTEFT as the donor. However, the origin of the improved performance compared to fullerenes is not well understood and EQE $>80 \%$ has not yet been achieved. The remarkably high $J_{\mathrm{sc}}$ and EQE of PBDTTT-EFT:EHIDTBR devices are investigated in detail in the following sections.

Origin of high photocurrent and external quantum efficiency. Figure 1c shows the EQEs of the PBDTTT-EFT:EHIDTBR (modified with PEIE and EA, $80 \mathrm{~nm}$ ) and the PBDTTT-EFT: $\mathrm{PC}_{71} \mathrm{BM}$ devices $(90 \mathrm{~nm})$. Figure $1 \mathrm{~d}$ shows the product of EQE and the Air Mass (AM)1.5G solar spectrum for the two EQEs determined experimentally compared to the $\mathrm{EQE}_{\mathrm{SQ}}$ in the Shockley-Queisser limit for a bandgap $\left(E_{\mathrm{g}}\right)$ of $1.6 \mathrm{eV}\left(\mathrm{EQE}_{\mathrm{SQ}}=1\right.$ 
a

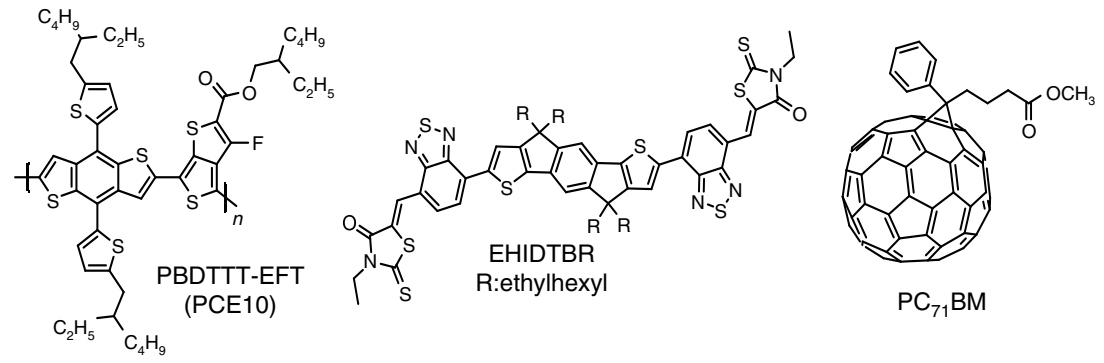
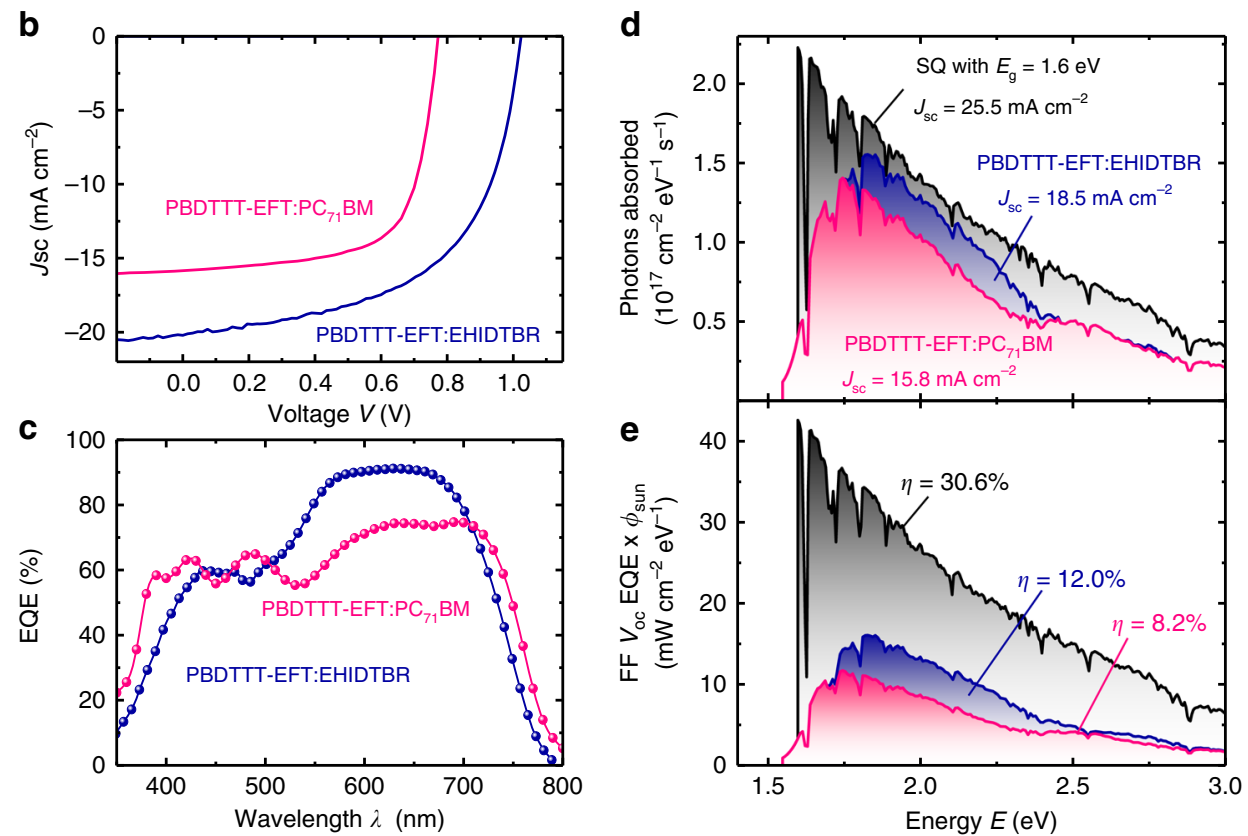

Fig. 1 Chemical structures and photovoltaic characteristics of PBDTTT-EFT devices. a Chemical structures of PBDTTT-EFT, EHIDTBR, and PC ${ }_{71} B M$. b Current-voltage characteristics of PBDTTT-EFT:EHIDTBR and PBDTTT-EFT:PC 71 BM devices under $100 \mathrm{~mW} \mathrm{~cm}^{-2}$ illumination. $\mathbf{c}$ Internal and external quantum efficiency spectra of the corresponding devices. $\mathbf{d}$ External quantum efficiency multiplied with the solar spectrum for the data from $\mathbf{c}$ and for a step-function-like quantum efficiency (Shockley-Queisser limit) for a bandgap of $1.6 \mathrm{eV}$. e Product of solar spectrum, quantum efficiency, fill factor, and open-circuit voltage for the three cases from $\mathbf{d}$. The area under the curves in e corresponds to a power density and is directly proportional to the efficiency of the devices

\section{Table 1 Key photovoltaic parameters of PBDTTT-EFT:PC ${ }_{71} B M$ and PBDTTT-EFT:EHIDTBR solar cell devices using different modified electron-transport layers}

\begin{tabular}{|c|c|c|c|c|}
\hline PBDTTT-EFT:EHIDTBR & $V_{\text {oc }}(\mathrm{V})$ & $J_{s c}\left(m A c m^{-2}\right)$ & $\mathbf{F F}$ & $\eta_{\max }(\%)$ (average \%) \\
\hline $\mathrm{ZnO}$ & $1.01( \pm 0.01)$ & $18.2( \pm 0.4)$ & $0.56( \pm 0.02)$ & $10.5(10.3)$ \\
\hline PEIE & $1.03( \pm 0.01)$ & $17.8( \pm 0.5)$ & $0.63( \pm 0.01)$ & 11.5 (11.3) \\
\hline $\mathrm{ZnO}+\mathrm{PEIE}+\mathrm{EA}(150 \mathrm{~nm})$ & $1.03( \pm 0.01)$ & $20.2( \pm 0.3)$ & $0.57( \pm 0.02)$ & $11.8(11.5)$ \\
\hline \multicolumn{5}{|c|}{ PBDTTT-EFT:PC ${ }_{71} B M$ (3\% DIO) } \\
\hline
\end{tabular}

for $E>E_{\mathrm{g}}$ and 0 for $E<E_{\mathrm{g}}$ ). The area under the curves correlates with $J_{\text {sc }}$. The PBDTTT-EFT:EHIDTBR cell achieves its high peak EQE by combining the absorption of donor and acceptor in the same photon energy region between 1.7 and $2.5 \mathrm{eV}$. Here, the PBDTTT-EFT:EHIDTBR-based device collects substantially more photons than the cell with $\mathrm{PC}_{71} \mathrm{BM}$. The loss in EQE at higher photon energies is less relevant because of the reduced photon flux in the solar spectrum for energies above $2.5 \mathrm{eV}$. Figure $1 \mathrm{e}$ combines the data in $1 \mathrm{~d}$ with the fill factor FF and the $V_{\text {oc }}$ values to achieve PCE as a function of energy. The area under the curves in Fig. 1e is now directly proportional to the power density at the maximum power point and therefore also solar cell efficiency. While the PBDTTT-EFT:EHIDTBR device is still superior relative to the fullerene-based device, now the relatively low FF (63\%) leads to substantial losses relative to the Shockley-Queisser limit, which has a FF around $90 \%$. This clearly highlights that there is 
a

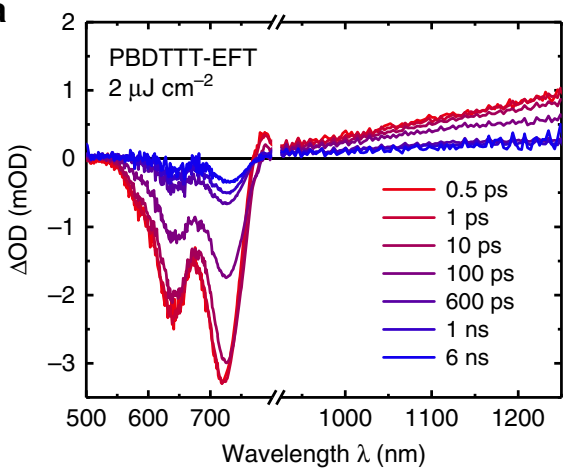

C

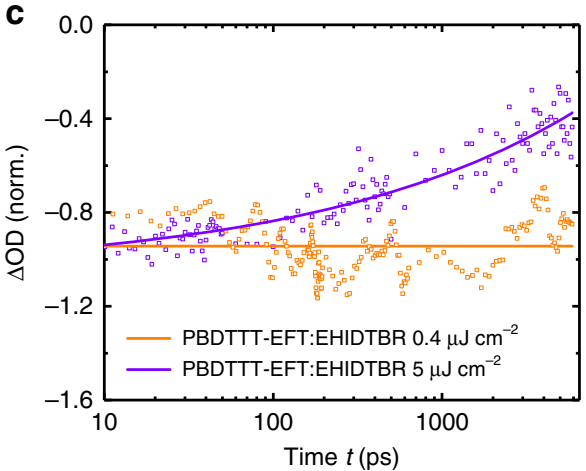

b

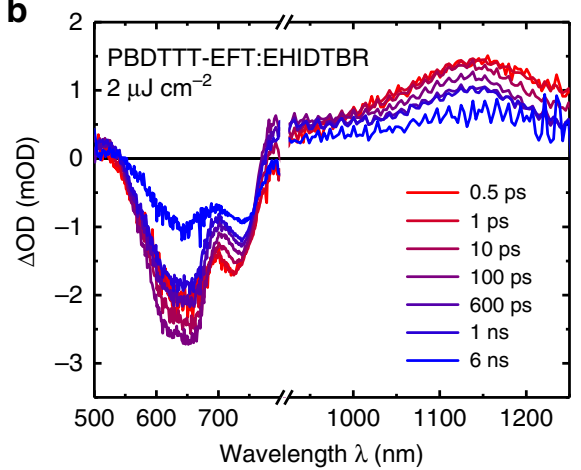

d

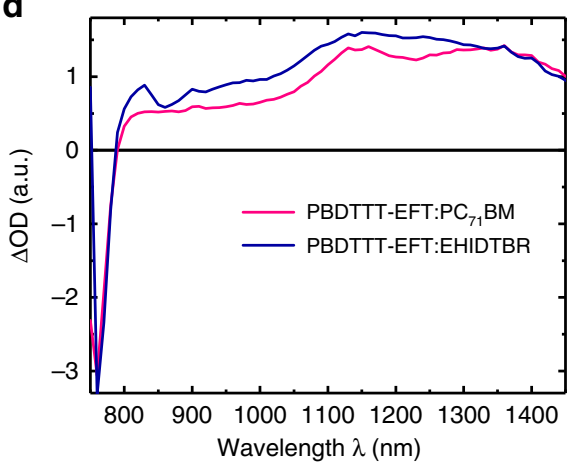

Fig. 2 Spectroscopy data showing fast exciton dissociation and efficient polaron formation. Transient absorption spectra for a (a) neat PBDTTT-EFT film and (b) PBDTTT-EFT:EHIDTBR blend film, following excitation of the PBDTTT-EFT at $500 \mathrm{~nm}$. Spectra are shown as a function of time delay after excitation from $0.5 \mathrm{ps}$ to $6 \mathrm{~ns}$. c The blend decay dynamics at $730 \mathrm{~nm}$ as a function of excitation density. At low excitation densities $\left(0.4 \mu \mathrm{J} \mathrm{cm}^{-2}\right)$, no decay is apparent, indicative of an absence of geminate recombination dynamics; at higher excitation densities $\left(5 \mu \mathrm{J} \mathrm{cm}{ }^{-2}\right)$, significant decay is observed on this timescale (up to 6 ns) indicative of bimolecular, non-geminate recombination losses. These non-geminate recombination losses are also apparent as an absorbance loss at long time delays in $\mathbf{b}$ (measured at $2 \mu \mathrm{cm}^{-2}$ ). $\mathbf{d}$ Photo-induced absorption spectra of PBDTTT-EFT:PC 71 BM and PBDTTT-EFT:EHIDTBR excited at $532 \mathrm{~nm}$ and recorded under vacuum of $10^{-6}$ Torr and a temperature $T=10 \mathrm{~K}$ illustrating the polaron yield of PBDTTT-EFT:EHIDTBR compared to PBDTTT-EFT:PC 71 BM

room for improvement for FF values, ideally for thicker devices to have even steeper absorption onsets that will boost EQEs in these systems.

Transient dynamics of the photo-generated excitons and polarons provide information on the losses of an active layer, which then can be correlated to the photocurrent generation ${ }^{31-34}$. Figure 2 shows $f s$-transient absorption spectra (TAS) for neat PBDTTT-EFT and blended PBDTTT-EFT:EHIDTBR films, employing $500 \mathrm{~nm}$ excitation to selectively excite the polymer in both films. Control data for EHIDTBR and PBDTTT-EFT: $\mathrm{PC}_{71} \mathrm{BM}$ are shown in Supplementary Fig. 1. Blending PBDTTTEFT with EHIDTBR or $\mathrm{PC}_{71} \mathrm{BM}$ results in the appearance of a long-lived, narrow photo-induced absorption band centered at $1150 \mathrm{~nm}$, assigned to polymer polaron absorption. The shape of this photo-induced absorption is clearly distinct from that of either PBDTTT-EFT or EHIDTBR excitons (both of which exhibit steadily increasing absorption from 850 to $1100 \mathrm{~nm}$, in contrast to the blend spectra). This polaron absorption appears for both blends from early times, with its spectral evolution being completed within $10 \mathrm{ps}$, indicating ultrafast charge separation on this timescale or faster (smaller than $10 \mathrm{ps}$ ). This suggests a blend morphology comprising primarily highly intermixed donor and acceptor molecules, enabling rapid exciton separation without requiring significant exciton diffusion. Photoluminescence quenching results supported these observations with strong photoluminescence (PL) quenching of EHIDTBR (99\%) and PBDTTT-EFT emission (91\%) in the blend relative to the neat films, indicative of efficient separation of both excitons (Supplementary Fig. 2). The lower photoluminescence quenching
(PLQ) for PBDTTT-EFT excitons most probably arises from the presence of a small fraction of pure polymer in domains with diameters similar to or greater than the exciton diffusion length observed also in X-ray diffraction (XRD) traces (Supplementary Fig. 3a). Time-resolved luminescence decay of the neat and blend films are in agreement with the PLQ data showing 91\% quenching when EHIDTBR is mixed with PBDTTT-EFT (1:1 w-w) (Supplementary Fig. 2i). Differential scanning calorimeter (DSC) traces reveal that neat EHIDTBR exhibit broad endothermic transitions at temperatures around $220^{\circ} \mathrm{C}$, attributed to the crystalline phase melt (Supplementary Fig. 3b). In contrast to EHIDTBR, no such thermally induced crystallization occurs during the heating cycle of PBDTTT-EFT due to its amorphous nature. The EHIDTBR crystalline transition in PBDTTT-EFT:EHIDTBR shows decreased enthalpy and minimized melting point depression. This implies that PBDTT-EFT partly diffuses into the EHIDTBR phase in the blend (Supplementary Fig. 3c). The cooling scan shows a reduced recrystallization, which can be described as having a mixed PBDTTTEFT:EHIDTBR region, comprising a crystalline acceptor phase and pure polymer domains, which reduces the PL quenching as well (Supplementary Fig. 2c, d).

It is also apparent from Fig. $2 \mathrm{~b}$ at longer time delays ( 10 ps to 6 $\mathrm{ns}$ ), the photo-induced absorption decays in amplitude, assigned to charge recombination losses. However, this was observed to be only present at high excitation densities, and was absent (within our signal to noise of $\pm 10 \%)$ at low excitation densities $\left(0.4 \mu \mathrm{J} \mathrm{cm}^{-2}\right)$ (Fig. 2c). We thus attribute the recombination losses observed at high laser intensities to non-geminate recombination and conclude that geminate (monomolecular) recombination losses are 

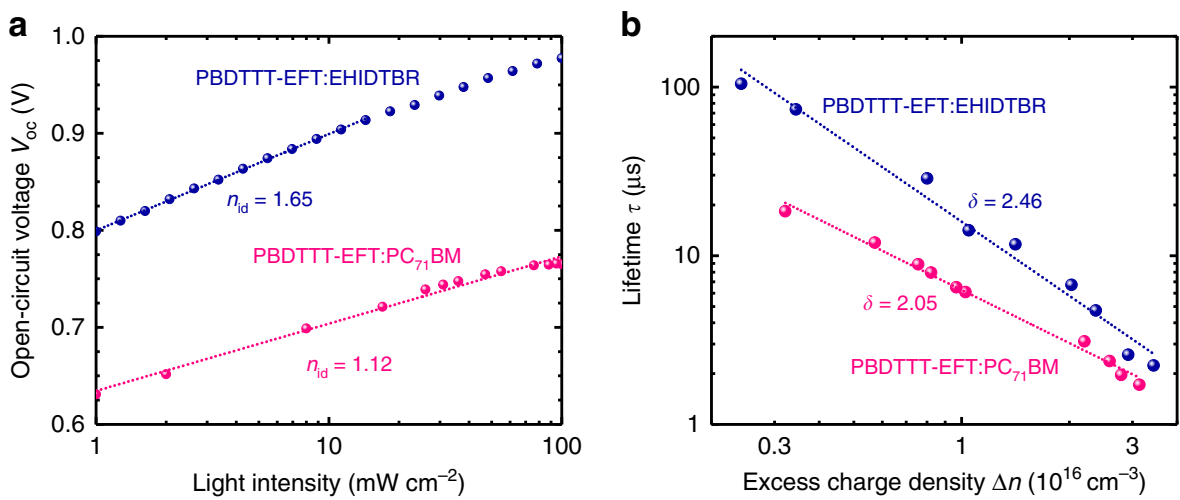

Fig. 3 Analysis of recombination mechanism and dynamics in PBDTTT-EFT-based solar cells. a $V_{\text {oc }}$ versus light intensity for PBDTTT-EFT:EH-IDTBR and PBDTTT-EFT:PC 71 BM devices. b Charge-carrier lifetime $\tau$, obtained from TPV, as a function of charge density $n$, calculated from CE measurements under $V_{\text {oc }}$ conditions (from 0.2 to 2 suns). The slope presents the recombination order $(\delta)$ of the studied systems. The dashed lines represent linear fits of the data

minimized in PBDTTT-EFT:EHIDTBR blend, at least over the timescale studied (we note these non-geminate losses were only observed under high laser excitation conditions, and are therefore not relevant to device performance under solar irradiance). Based on these results, it is clear that polymer exciton separation leads to almost complete charge dissociation, with a negligible $(<10 \%)$ yield of geminate recombination from any bound "charge transfer" or "polaron pair" states formed at the donor/acceptor interface. The absence of geminate recombination losses combined with efficient exciton separation is likely to be a key reason behind the remarkably high $J_{\mathrm{sc}}$ and IQE (EQE/absorptance) data we report herein for PBDTTT-EFT:EHIDTBR solar cells (Supplementary Fig. 2e-h). The average IQE for the PBDTTTEFT:EHIDTBR blend is above 95\% throughout the visible region between 550 and $700 \mathrm{~nm}$. The absence of geminate recombination losses in these devices, in contrast to, for example, the PffBT4T-2DT:FBR devices we have reported previously ${ }^{16}$, most probably results from the larger energy offsets driving exciton separation in the PBDTTT-EFT:EHIDTBR blend system (0.05 versus $0.25 \mathrm{eV}$, respectively.) To further elucidate the reasons for the enhanced $J_{\mathrm{sc}}$ of PBDTTT-EFT:EHIDTBR blend compared to PBDTTT-EFT:PC ${ }_{71} \mathrm{BM}$, we used photo-induced absorption spectroscopy (PIA) ${ }^{35}$. By employing this technique, we can directly measure the concentration of long-lived photo-generated charge carriers in the blend ${ }^{36}$. Figure $2 \mathrm{~d}$ depicts the PIA traces of PBDTTT-EFT blended with the two acceptors at $10 \mathrm{~K}$. Notably, in agreement with the higher $J_{\mathrm{sc}}$ obtained for EHIDTBR-based solar cells, we found that the absorption feature at $1150 \mathrm{~nm}$ is considerably higher for the PBDTTT-EFT:EHIDTBR blend indicating larger polaron yield and/or longer polaron lifetimes compared to the $\mathrm{PC}_{71} \mathrm{BM}$ blend.

Taking into account the photovoltaic parameter evolution of PBDTTT-EFT:PC ${ }_{71} \mathrm{BM}$ devices compared to PBDTTT-EFT: EHIDTBR cells from Table 1, it is clear that the limiting factor for PBDTTT-EFT:EHIDTBR device efficiencies beyond $12 \%$ is the fill factor values around 0.63 . The FF can be expressed as the compromise between recombination and extraction of charge carriers $^{37}$. As aforementioned, PBDTTT-EFT:EHIDTBR blend lacks geminate recombination, thus we focus on gaining insight into non-geminate recombination from solar cell devices. We first investigate the photo-induced charge-carrier mobility $(\mu)$ of the devices by employing photo-induced charge-carrier extraction by linearly increasing voltage (photo-CELIV). The photocurrent traces of PBDTTT-EFT:PC ${ }_{71} \mathrm{BM}$ and PBDTTT-EFT:EHIDTBR solar cells (Supplementary Fig. 4) clearly reveal that the $t_{\max }$ has nearly identical for the two devices $\left(\mu\right.$ of $2.1 \times 10^{-4} \mathrm{~cm}^{2} \mathrm{~V}^{-1} \mathrm{~s}^{-1}$ and $2.0 \times 10^{-4} \mathrm{~cm}^{2} \mathrm{~V}^{-1} \mathrm{~s}^{-1}$ for PBDTTT-EFT:PC 71 BM and PBDTTT-EFT:EHIDTBR devices, respectively). The closely similar charge-carrier mobility suggests that this is not the cause of the lower FF for the PBDTTT-EFT:EHIDTBR devices. As such, we carried out additional current-voltage characteristics and transient measurements for the analysis of recombination and lifetime of charge carriers in PBDTTT-EFT:EHIDTBR solar cells. An initial test of the dominant recombination mechanism in a solar cell already follows from dependence of the $V_{\mathrm{oc}}$ on the light intensity, $\Phi_{\text {in }}{ }^{38}$. One can express the $V_{\text {oc }}$ as $V_{\text {oc }}=n_{\text {id }} k T / q \ln \left(\Phi_{\text {in }}\right)+$ const, where $n_{\text {id }}$ is the ideality factor and $k T / q$ is the thermal voltage. Values of $n_{\mathrm{id}}$ of 1 in a device with a fully depleted absorber can be assigned either to surface recombination or to direct recombination between electrons and holes, while $n_{\mathrm{id}}>1$ indicates localized states in the bandgap being involved in recombination. As depicted in Fig. 3a, we obtain ideality factors of 1.15 and 1.65 for the PBDTTT-EFT:PC ${ }_{71} B M$ and PBDTTT-EFT:EHIDTBR devices, respectively. This suggests that shallow trap states are involved in recombination in case of the EHIDTBR-based solar cells, which likely contributes to the decreased FF and is consistent with higher $n_{\text {id }}$ obtained in other low-FF NFA-based devices ${ }^{16}$.

In parallel, we investigated the recombination mechanisms in more detail. As such, the methods transient photovoltage (TPV) and charge extraction (CE) has been studied to calculate the charge-carrier lifetime $(\tau)$ and excess charge-carrier density $(\Delta n)$ ), respectively. Figure $3 \mathrm{~b}$ shows the TPV lifetime as a function of the charge-carrier density that was extracted at the same light intensity, when switching from open circuit to short circuit while switching off the bias light at the same time. At a given extracted charge density $\Delta n$, the PBDTTT-EFT:EHIDTBR-based solar cell has a higher lifetime, which contributes to the enhanced opencircuit voltage of the device. From the slope of lifetime or recombination rate $R$ versus charge-carrier density, we can determine the reaction order $\delta$, defined as the exponent in the relation $R \sim \Delta n^{\delta}$. For the $\mathrm{PC}_{71} \mathrm{BM}$-based solar cell, the reaction order is close to 2 and the charge density scales with voltage with $\Delta n \sim \exp \left(q V_{\mathrm{oc}} / 2 k T\right)$ (Supplementary Fig. 5). NFA-based device has both higher ideality factors and higher reaction orders. In addition, charge density scales approximately with $\Delta n \sim \exp$ $\left(q V_{\text {oc }} / 3.3 k T\right)$ (Supplementary Fig. 5), which also suggests the existence of localized shallow trap states in the bandgap ${ }^{39}$. It appears that the PBDTTT-EFT:EHIDTBR device offers more degrees of freedom and also more energetic disorder, which could limit the $\mathrm{FF}$ values to $65 \%^{39}$. 

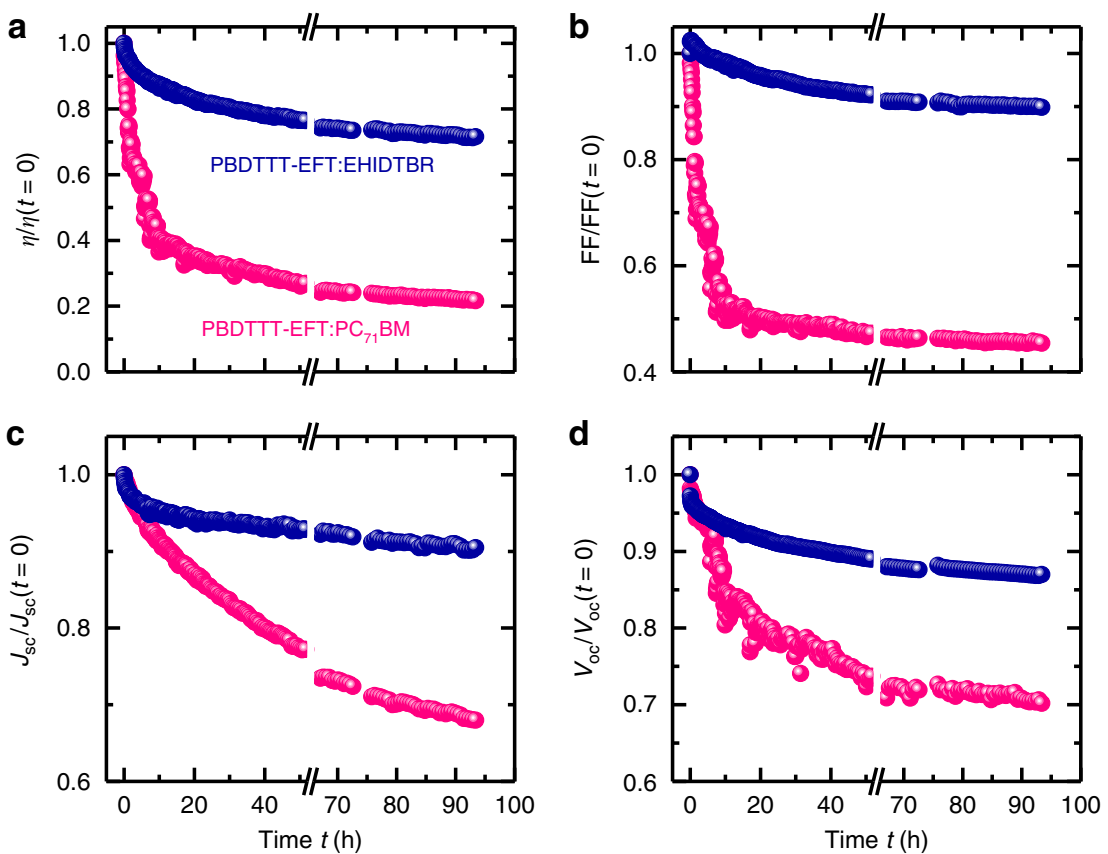

Fig. 4 Stability data for fullerene and nonfullerene devices. Time evolution of device performance and figures of merit of PBDTTT-EFT:EHIDTBR and PBDTTT-EFT:PC ${ }_{71}$ BM solar cell devices under illumination of metal halide lamp in $\mathrm{N}_{2}$ atmosphere

Influence of phase separation on lifetime and degradation. A robust nano-morphology against irradiation is required for long lifetime solar cells. There have been efforts for such a nanomorphology in Organic solar cells (OSCs) using molecular locks or alloy acceptors in fullerene solar cells ${ }^{40,41}$; however, the highefficiency devices usually suffered from optimization using solvent additives that cause demixing of the polymer:fullerene blend $^{42-44}$. Recently, NFA devices outperformed fullerene device stabilities with interface engineering using low-temperature $\mathrm{TiO}_{x}$ for conventional structures ${ }^{45,46}$; nevertheless a recipe for a robust nano-morphology of the polymer-NFA blend is missing and has not been explored yet. To study the severity of degradation, both PBDTTT-EFT:PC ${ }_{71}$ BM and PBDTTT-EFT:EHIDTBR devices were in an environmentally controlled chamber $\left(\mathrm{O}_{2}\right.$ and $\mathrm{H}_{2} \mathrm{O}$ lower than 1 p.p.m.) and the photovoltaic parameters were probed simultaneously under a metal halide lamp (irradiated at 100 $\mathrm{mW} \mathrm{cm}{ }^{-2}$ ). Figure 4 summarizes the time evolution of photovoltaic parameters of both devices for up to $100 \mathrm{~h}$ of degradation. $\mathrm{PC}_{71}$ BM-based PBDTTT-EFT devices suffer from an abrupt decrease in FF and thus efficiency even after $24 \mathrm{~h}$, whereas there is only a gradual decrease in efficiency (around 20\%) for the EHIDTBR device over a period of $100 \mathrm{~h}$, which is similar to other NFA systems $s^{7,13,47}$. Generally, for commercial application, ultraviolet (UV) filter can be adopted to avoid the negative impact of UV light on the device stability. Thus, we replaced the metal halide lamp (MHI) lamps white LED lights ${ }^{48}$. As a result, PBDTTT-EFT:EHIDTBR cells show high stability (PCE drop $<10 \%$ ) up to $1000 \mathrm{~h}$ (Supplementary Fig. 7), which is a benchmark for long-term stability. Dynamic secondary ion mass spectrometry (SIMS) was used to understand the depth profile of both blends and explain the huge difference in stability with NFA devices. The advantage of SIMS relies on its capability to track the elemental distribution in depth allowing us to obtain valuable information regarding the homogeneity and miscibility of the donor and acceptor across the active layer ${ }^{28,49}$. The SIMS data in Fig. 5 illustrate the distribution of elements and ions as a function of depth within the PBDTTT-EFT:PC ${ }_{71} B M$ and PBDTTT-EFT: EHIDTBR blends spin-cast on zinc oxide $(\mathrm{ZnO}) / \mathrm{Si}$ substrates. We compared SIMS data obtained from fresh samples (prior to light radiation) to those acquired for samples subjected to metal halide light radiation (equivalent to $24 \mathrm{~h}$ ). In order to identify the signature of each component in both blends, the fluorine element described by the $\mathrm{F}^{-}$signal is ascribed to PBDTTT-EFT, whereas the EHIDTBR is tracked by following the $\mathrm{CN}^{-}$signal. Although the $\mathrm{PC}_{71} \mathrm{BM}$ is composed of $\mathrm{C}, \mathrm{O}$, and $\mathrm{H}$, which are also present in the PBDTTT-EFT polymer, its behavior may be examined by following $\mathrm{C}^{-}$and $\mathrm{C}_{3}{ }^{-}$signals as will be discussed below. Assuming the aforementioned assignments, the SIMS data shows clearly that the PBDTTT-EFT:EHIDTBR blend has a very similar depth profile before and after light radiation. The elemental depth distribution of the $\mathrm{F}^{-}$and $\mathrm{CN}^{-}$signals indicates a homogeneous distribution of both PBDTTT-EFT and EHIDTBR across the active layer. It should be noted that the increase of all ion signals at the $\mathrm{ZnO}$ interface is an artefact due to a change of the chemical environment. In contrast to PBDTTT-EFT:EHIDTBR, the PBDTTT-EFT:PC ${ }_{71} \mathrm{BM}$ mixture prepared with $3 \%$ DIO represents a pronounced vertical phase separation and demixing following the light radiation. These observations were also in line with the high-resolution transmission electron microscopy of the active layer blends. (Supplementary Fig. 6) PBDTTT-EFT, denoted by $\mathrm{F}^{-}$and $\mathrm{C}_{2} \mathrm{H}_{2}^{-}$is clearly pushed toward the $\mathrm{ZnO}$ interface. However, $\mathrm{C}^{-}$and $\mathrm{C}_{3}{ }^{-}$signals are more concentrated in the first $50 \mathrm{~nm}$ depth beyond which they exhibit a clear decrease throughout the active layer, which is more likely to represent the fullerene acceptor. Thus fullerene acceptors are located closer to the surface than $\mathrm{ZnO}$ when the PBDTTT-EFT:PC ${ }_{71} \mathrm{BM}$ blend is subjected to light radiation due to demixing that results in severe reduction in FF values. We believe that this is due to high boiling point solvent additive (DIO) used to optimize the morphology of fullerene devices. A similar meta-stable morphology observation was reported by Li et al. ${ }^{42}$, where DIO had effect not only in longterm lifetime of the devices but also in the initial measurements as burn-in that causes spinodal demixing. These results suggest that high-efficiency solar cell devices optimized with solvent additives would not be the choice for long-term stable commercial applications. 

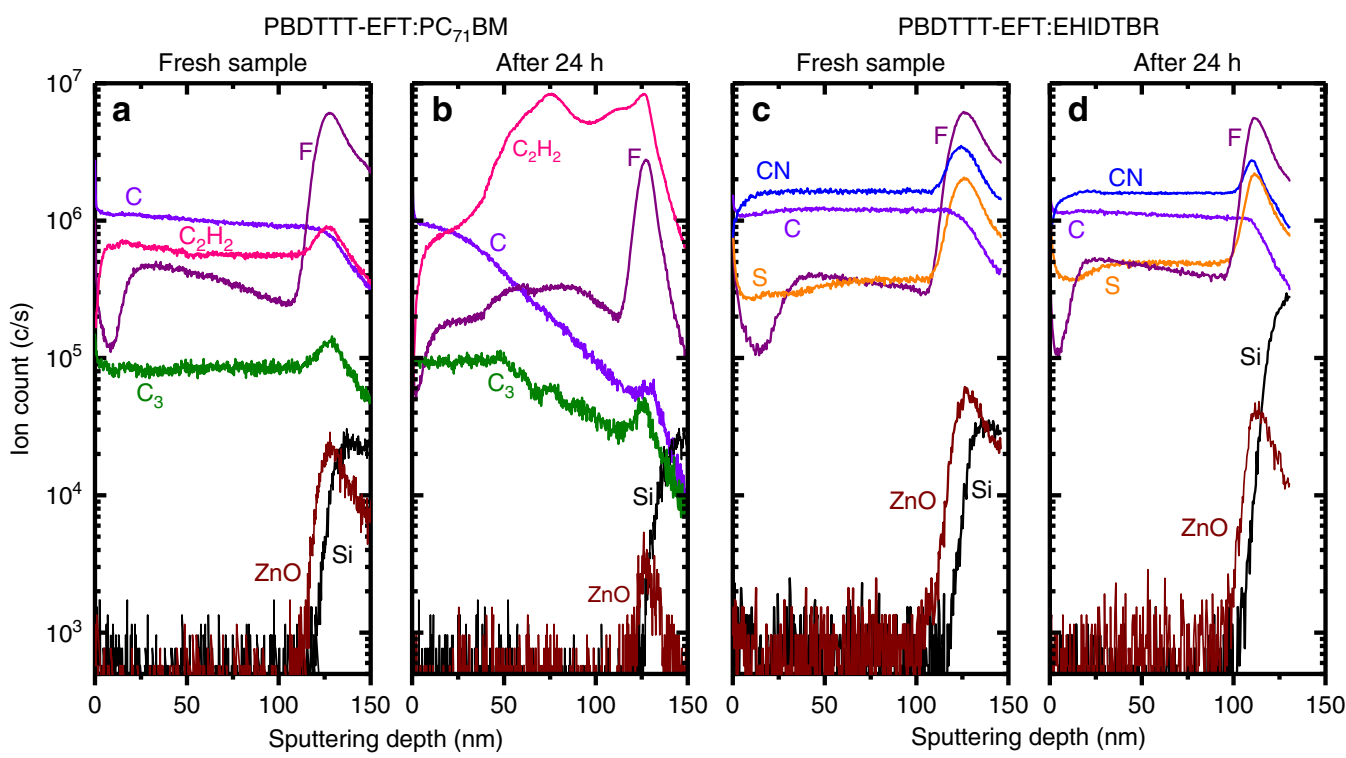

Fig. 5 Depth profiles before and after degradation. Negative SIMS depth profiling of PBDTTT-EFT:PC 71 BM (a, b) and PBDTTT-EFT:EHIDTBR (c, d) blends prior to light radiation and subjected to light radiation equivalent to $24 \mathrm{~h}$. $0 \mathrm{~nm}$ sputtering depth represents the top surface and $150 \mathrm{~nm}$ shows the substrate $(\mathrm{ZnO})$

In conclusion, we report a high-performance organic photovoltaics (OPV) system based on a small-molecule acceptor that delivers a PCE of $12 \%$ with an EQE around $90 \%$ and a $V_{\text {oc }}$ of 1.03 $\mathrm{V}$. We elucidate that the concomitant effect of reduced geminate recombination and efficient charge separation leads to an exceptionally high $J_{\mathrm{sc}}$ of $20 \mathrm{~mA} \mathrm{~cm}^{-2}$. In parallel to the optoelectronic analysis, we demonstrate that the NFA cells depict a more robust microstructure toward light degradation compared to the fullerene counterparts. Most obviously, the LUMO level offset between PBDTTT-EFT and EHIDTBR is still sufficient to drive efficient exciton separation and charge dissociation. It is also surprising that this blend consisting of an amorphous donor does not foster geminate losses but yields charges efficiently. One of the possible explanations is that, as illustrated from the DSC, TEM, PLQ, and XRD data, a highly intermixed region of PBDTTT-EFT and EHIDTBR ensures efficient exciton dissociation. The larger pure domains of PBDTTT-EFT and crystalline acceptor phase of EHIDTBR (from DSC) enables the spatial separation of photo-generated carriers. In addition, an efficient charge separation is enabled by sufficiently large energetic offset and suppress geminate recombination losses. Overcoming trapassisted recombination remains a challenge for organic solar cells and an elegant approach to overcome this could be ternary blend, in which a more ordered polymer or small molecule is added to a binary system to facilitate charge transport, which has been shown to result in a higher $\mathrm{FF}^{28,50}$. Overall, these results suggest that overcoming trap-assisted recombination, i.e., reducing the defects, by tackling FF losses with further interface engineering creates the opportunity for durable OPV systems for commercialization.

\section{Methods}

Materials. PBDTTT-EFT was purchased from Cal-Os chemicals. EHIDTBR was synthesized according to the previous reports ${ }^{13}$. $\mathrm{PC}_{71} \mathrm{BM}(99.5 \%)$ was purchased from Solenne BV. All the other materials and solvents are received from SigmaAldrich and used without further purification.

Device fabrication and characterization. The inverted architecture devices were processed in inert atmosphere using ITO/ETL/active layer/ $\mathrm{MoO}_{3}(10 \mathrm{~nm}) / \mathrm{Ag}(100$ $\mathrm{nm}$ ) stack, where ETL were zinc oxide (using sol-gel method) and ethoxylated PEIE, active layers were PBDTTT-EFT:EHIDTBR (1:2.5, w-w\%) and PBDTTTEFT:PC ${ }_{71} \mathrm{BM}(1: 1,5, \mathrm{w}-\mathrm{w} \%)$. Cleaning of the pre-structured ITO substrates has been done using deionized water, acetone, and isopropyl alcohol (10 min each). Eight minutes of plasma treatment was applied to treat the substrates. A thin layer of $\mathrm{ZnO}$ was deposited from a precursor solution of zinc acetatedihydrate $(2 \mathrm{ml} \mathrm{2}$ methoxyethanol and $60 \mu \mathrm{L}$ ethanolamine). A 30-40 nm ZnO layer was formed after $20-25 \mathrm{~min}$ annealing at $150^{\circ} \mathrm{C}$. The modification of $\mathrm{ZnO}$ layer was done with either spin-coating a $1 \%$ ethanolamine in 2-methoxyethanol on existing annealed $\mathrm{ZnO}$ layers followed by annealing at $100^{\circ} \mathrm{C}$ for $10 \mathrm{~min}$, spin-casting PEIE solution in ethanol using 4000 r.p.m., and annealing at $80^{\circ} \mathrm{C}$ for $10 \mathrm{~min}$ or both procedures following the consecutive order on top of $\mathrm{ZnO}$ layer $(\mathrm{ZnO}+\mathrm{PEIE}+\mathrm{EA})$. Active layers were spin-cast from $24 \mathrm{mg} \mathrm{ml}^{-1}$ concentrated solutions in chlorobenzene to result in $90-100 \mathrm{~nm}$ thick layers. Thick devices were prepared using $28 \mathrm{mg} \mathrm{ml}^{-1}$ total solution. $\mathrm{MoO}_{3}(10 \mathrm{~nm})$ and silver $(100 \mathrm{~nm})$ were evaporated using a shadow mask (with an aperture of $\left.0.045 \mathrm{~cm}^{2}\right)$ under vacuum $\left(1 \times 10^{-6} \mathrm{mbar}\right)$. No post annealing was performed to any of the devices. $J-V$ characteristics were measured under dark and illumination using a xenon lamp from oriel Instruments (AM 1.5 $\mathrm{G}, 100 \mathrm{~mW} \mathrm{~cm}^{-2}$ ). EQE spectra were obtained using a silicon photodiode reference with $100 \mathrm{~W}$ Tungsten-Halogen lamp. Six pixels are produced for averaging the results.

Photo-CELIV measurements. Photo-CELIV measurements were performed with a $405 \mathrm{~nm}$ laser-diode. An internal $50 \Omega$ resistor is used with an oscilloscope (Agilen technologies DSO-X 2024A) to record the current transients. A fast electrical switch was used to isolate the cell and prevent CE or sweep out during the laser pulse and the delay time. After a variable delay time, a linear extraction ramp was applied via a function generator. The ramp, which was $60 \mu$ s long and $2 \mathrm{~V}$ in amplitude, was set to start with an offset matching the $V_{\mathrm{oc}}$ of the cell for each delay time.

TPV and CE measurements. Solar cells were brought to $V_{\text {oc }}$ condition with a 405 $\mathrm{nm}$ laser-diode. The light intensity is adjusted over a range of 0.2 to 2 suns (with less than $0.5 \%$ error) by controlling the waveform generator (Agilent $33500 \mathrm{~B}$ ) and the intensity of a linear photodiode. A second $405 \mathrm{~nm}$ laser is used to induce a small perturbation in the device by a function generator (Agilent). The short laser pulse intensity ( $50 \mathrm{~ns}$ ) was used to adjust the voltage perturbation below $10 \mathrm{mV}$ (about $5 \mathrm{mV}$ ). The voltage decays to initial steady state after the pulse in a single exponential decay. The times of the decay was determined using a linear fit to the log plot of the voltage transient and small perturbation charge-carrier lifetime. In CE studies, the $405 \mathrm{~nm}$ laser pulsed the solar cells for $200 \mu$ s to bring the devices to a constant open-circuit voltage condition. Once the illumination stops, an analog switch was triggered to bring the solar device to Jsc condition $(50 \Omega)$ with $<50 \mathrm{~ns}$.

Transient absorption spectroscopy (fs-TAS). TAS measurements were performed by first generating a $800 \mathrm{~nm}$ laser pulse $(1 \mathrm{kHz}, 90 \mathrm{fs})$ from a commercial Solstice (Newport Corporation) Ti:sapphire regenerative amplifier. A part of the laser pulse optically directed through a TOPAS-Prime (light conversion) optical parametric amplifier to generate a $500 \mathrm{~nm}$ excitation pump laser. The other part of the laser output was used to generate the probe light in visible/near-IR continuum by sapphire crystals $(0.4$ and $1 \mathrm{~cm}$ thickness, respectively). The spectra and decays 
were obtained by a HELIOS transient absorption spectrometer (500-1300 nm) to 6 ns. The samples were measured in $\mathrm{N}_{2}$ atmosphere.

Photo-induced absorption measurements. Photo-induced absorption (PIA) studies were performed by exciting the sample with a $405 \mathrm{~nm}$ laser while simultaneously probing the sample with a white lamp. The PIA spectra of the sample were dispersed by a 1200 lines per mm grating monochromator (iHR320) and detected by a silicon detector through lock-in technique.

Ageing and lifetime measurements. The photovoltaic devices are constructed in the same way described in the device characterization part. The moisture and $\mathrm{O}_{2}$ concentrations were kept below 0.5 p.p.m. for the ageing experiments in $\mathrm{N}_{2}$ atmosphere in a closed chamber system. Metal halide lamp with UV part is used to irradiate the samples (T: 30 to $33^{\circ} \mathrm{C}$ ) for $24 \mathrm{~h}$ and 1 week for long-term degradation.

Dynamic secondary ion mass spectrometry. Depth-profiling experiments were performed on a dynamic SIMS instrument from Hiden analytical company (Warrington-UK) operated under ultra-high vacuum conditions, typically $10^{-9}$ torr. A continuous $\mathrm{Ar}^{+}$beam of $2 \mathrm{keV}$ energy was employed to sputter the surface while the selected ions were sequentially collected using a MAXIM spectrometer equipped with a quadrupole analyzer. In order to avoid the edge effect, the SIMS data were extracted from the acquisition area of $50 \times 50 \mu \mathrm{m}^{2}$ centered in the middle of the sputtered area estimated to be $500 \times 500 \mu \mathrm{m}^{2}$ using an adequate electronic gating. Assuming a constant sputtering rate, the conversion of timescale to depth scale was carried out by measuring the depth of the crater generated at the end of the depth-profiling experiment using a stylus profiler from Veeco.

Transmission electron microscopy. Dark-field transmission electron microscopy (TEM) images of bulk heterojunction (BHJ) films for morphological characterization were performed with a FEI Titan 80-300 TEM equipped with an electron monochromator, a Gatan imaging filter (GIF) Quantum 966 and a Cs probe corrector. All images were obtained in scanning TEM mode at $80 \mathrm{kV}$. Films were deposited on a PEDOT:PSS sacrificial layer in the following configuration PEDOT: $\mathrm{PSS} / \mathrm{ZnO} / \mathrm{BHJ}$ and they were then emerged in deionized water. Floating films of $\mathrm{ZnO} / \mathrm{BHJ}$ were collected with carbon-coated gold grids from Electron Microscopy Sciences. Films were then dried overnight in a vacuum chamber to remove any water residues.

X-ray diffraction measurement. X-ray diffraction measurement (XRD) measurement was conducted on PBDTTT-EFT and EHIDTBR neat and blend films, which were prepared by drop-casting about $0.25 \mathrm{ml}$ of solutions onto $2 \mathrm{~cm} \times 1 \mathrm{~cm}$ substrates and left to dry overnight at room temperature. The XRD is equipped with a PANALYTICAL X'PERT-PRO MRD diffractometer using a nickel-filtered $\mathrm{Cu} \mathrm{K}$ al beam and a X'CELERATOR detector, employing a current $I=40 \mathrm{~mA}$ and an accelerating voltage $U=40 \mathrm{kV}$.

Differential scanning calorimetry. Differential scanning calorimetry (DSC) experiments were carried out with a TA Instruments DSC Q20 at a heating rate of $5{ }^{\circ} \mathrm{C}$ per min under nitrogen; heating (and cooling) cycles were recorded. Samples were prepared by drop-casting the materials from chlorobenzene solution onto glass slides and allowing the solvent to evaporate under ambient conditions. The dry films were then scrapped off and transferred into DSC pans.

Time-resolved photoluminescence. Time-resolved photoluminescence (TRPL) spectra were recorded using a high-resolution streak camera system (Hamamatsu C10910) where the excitation beam is adjusted to $650 \mathrm{~nm}$ using Spectra Physics MaiTai eHP from a mode-locked Ti:sapphire laser (Coherent Mira 900D). During the measurements, emission of the samples was detected by a monochromator attached to a Universal Hamamatsu C6860 streak camera with a temporal resolution of $4 \mathrm{ps}$.

Ellipsometry measurements. For ellipsometry measurements, PBDTTT-EFT:EHIDTBR was spin coated onto silicon wafers coated with $25 \mathrm{~nm}$ of $\mathrm{SiO}_{2}$. The J.A. Woollam M2000 ellipsometer was used to collect the raw $\psi$ and $\Delta$ values that describe polarization change over the visible range. The $n, k$ values were obtained using Complete EASE software. Models were developed and fitted to the $\psi$ and $\Delta$ data until a suitable fit was found, indicated by a mean squared error (MSE) value $<2$.

Data availability. The data that support the findings of this study are available on request from the corresponding author (D.B.).

Received: 6 July 2017 Accepted: 15 April 2018

Published online: 25 May 2018

\section{References}

1. Vezie, M. S. et al. Exploring the origin of high optical absorption in conjugated polymers. Nat. Mater. 15, 746-753 (2016).

2. Alsulami, Q. A. et al. Remarkably high conversion efficiency of inverted bulk heterojunction solar cells: from ultrafast laser spectroscopy and electron microscopy to device fabrication and optimization. Adv. Energy Mater. 6, 1502356-1502363 (2016).

3. Nielsen, C. B., Holliday, S., Chen, H.-Y., Cryer, S. J. \& McCulloch, I. Nonfullerene electron acceptors for use in organic solar cells. Acc. Chem. Res. 48, 2803-2812 (2015).

4. Li, S. et al. Energy-level modulation of small-molecule electron acceptors to achieve over $12 \%$ efficiency in polymer solar cells. Adv. Mater. 28, 9423-9429 (2016).

5. Yao, H. et al. Achieving highly efficient nonfullerene organic solar cells with improved intermolecular interaction and open-circuit voltage. Adv. Mater. 29, 1700254-1700262 (2017).

6. Zhao, F. et al. Single-junction binary-blend nonfullerene polymer solar cells with $12.1 \%$ efficiency. Adv. Mater. 29, 1700144-1700151 (2017).

7. Baran, D. et al. Reducing the efficiency-stability-cost gap of organic photovoltaics with highly efficient and stable small molecule acceptor ternary solar cells. Nat. Mater. 16, 363-369 (2017).

8. Li, X., Liu, X., Zhang, W., Wang, H.-Q. \& Fang, J. Fullerene-free organic solar cells with efficiency over $12 \%$ based on EDTA-ZnO hybrid cathode interlayer. Chem. Mater. 29, 4176-4180 (2017).

9. Li, H. et al. Beyond fullerenes: design of nonfullerene acceptors for efficient organic photovoltaics. J. Am. Chem. Soc. 136, 14589-14597 (2014).

10. Fang, Y. et al. A narrow optical gap small molecule acceptor for organic solar cells. Adv. Energy Mater. 3, 54-59 (2013).

11. Jung, J. W. \& Jo, W. H. Low-bandgap small molecules as non-fullerene electron acceptors composed of benzothiadiazole and diketopyrrolopyrrole for all organic solar cells. Chem. Mater. 27, 6038-6043 (2015)

12. Liu, F. et al. Efficient semitransparent solar cells with high NIR responsiveness enabled by a small-bandgap electron acceptor. Adv. Mater. 29, 1606574-1606581 (2017).

13. Holliday, S. et al. High-efficiency and air-stable P3HT-based polymer solar cells with a new non-fullerene acceptor. Nat. Commun. 7, 11585 (2016).

14. Yao, H. et al. Design, synthesis, and photovoltaic characterization of a small molecular acceptor with an ultra-narrow band gap. Angew. Chem. Int. Ed. Engl. 56, 3045-3049 (2017).

15. Bin, H. et al. Non-fullerene polymer solar cells based on alkylthio and fluorine substituted 2D-conjugated polymers reach $9.5 \%$ efficiency. J. Am. Chem. Soc. 138, 4657-4664 (2016).

16. Baran, D. et al. Reduced voltage losses yield $10 \%$ efficient fullerene free organic solar cells with $>1 \mathrm{~V}$ open circuit voltages. Energy Environ. Sci. 9, 3783-3793 (2016).

17. Liu, J. et al. Fast charge separation in a non-fullerene organic solar cell with a small driving force. Nat. Energy 1, 16089 (2016).

18. Holliday, S. et al. A rhodanine flanked nonfullerene acceptor for solutionprocessed organic photovoltaics. J. Am. Chem. Soc. 137, 898-904 (2015).

19. Zhang, S., Ye, L. \& Hou, J. Breaking the $10 \%$ efficiency barrier in organic photovoltaics: morphology and device optimization of well-known PBDTTT polymers. Adv. Energy Mater. 6, 1502529-1502549 (2016).

20. Liu, S. et al. Thieno[3,4-c]pyrrole-4,6-dione-3,4-difluorothiophene polymer acceptors for efficient all-polymer bulk heterojunction solar cells. Angew. Chem. Int. Ed. Engl. 55, 12996-13000 (2016).

21. Deshmukh, K. D. et al. Critical role of pendant group substitution on the performance of efficient all-polymer solar cells. Chem. Mater. 29, 804-816 (2017).

22. Lee, C. et al. High-performance all-polymer solar cells via side-chain engineering of the polymer acceptor: the importance of the polymer packing structure and the nanoscale blend morphology. Adv. Mater. 27, 2466-2471 (2015).

23. Jung, J. W. et al. Fluoro-substituted n-type conjugated polymers for additivefree all-polymer bulk heterojunction solar cells with high power conversion efficiency of 6.71\%. Adv. Mater. 27, 3310-3317 (2015).

24. Mori, D., Benten, H., Okada, I., Ohkita, H. \& Ito, S. Highly efficient chargecarrier generation and collection in polymer/polymer blend solar cells with a power conversion efficiency of 5.7\%. Energy Environ. Sci. 7, 2939-2943 (2014).

25. Zhang, G. et al. Efficient nonfullerene polymer solar cells enabled by a novel wide bandgap small molecular acceptor. Adv. Mater. 29, 1606054-1606061 (2017).

26. Zhong, Y. et al. Molecular helices as electron acceptors in high-performance bulk heterojunction solar cells. Nat. Commun. 6, 8242 (2015).

27. Wu, Q., Zhao, D., Schneider, A. M., Chen, W. \& Yu, L. Covalently bound clusters of alpha-substituted PDI-rival electron acceptors to fullerene for organic solar cells. J. Am. Chem. Soc. 138, 7248-7251 (2016). 
28. Gasparini, N. et al. Designing ternary blend bulk heterojunction solar cells with reduced carrier recombination and a fill factor of $77 \%$. Nat. Energy 1, 16118 (2016)

29. He, Z. et al. Single-junction polymer solar cells with high efficiency and photovoltage. Nat. Photon. 9, 174-179 (2015).

30. Baran, D. et al. Facile synthesis and photovoltaic applications of a new alkylated bismethano fullerene as electron acceptor for high open circuit voltage solar cells. RSC Adv. 5, 64724-64730 (2015).

31. Dimitrov, S. et al. Singlet exciton lifetimes in conjugated polymer films for organic solar cells. Polymers 8, 1-12 (2016).

32. Schubert, M. et al. Correlated donor/acceptor crystal orientation controls photocurrent generation in all-polymer solar cells. Adv. Funct. Mater. 24 4068-4081 (2014).

33. Foertig, A. et al. Nongeminate and geminate recombination in PTB7:PCBM solar cells. Adv. Funct. Mater. 24, 1306-1311 (2014).

34. Jakowetz, A. C. et al. What controls the rate of ultrafast charge transfer and charge separation efficiency in organic photovoltaic blends. J. Am. Chem. Soc. 138, 11672-11679 (2016).

35. Min, J. et al. Two similar near-infrared (IR) absorbing benzannulated AzaBODIPY dyes as near-IR sensitizers for ternary solar cells. ACS Appl. Mater. Interfaces 5, 5609-5616 (2013).

36. Gasparini, N. et al. Organic solar cells: an alternative strategy to adjust the recombination mechanism of organic photovoltaics by implementing ternary compounds. Adv. Energy Mater. 5, 1501527-1501534 (2015).

37. Bartesaghi, D. et al. Competition between recombination and extraction of free charges determines the fill factor of organic solar cells. Nat. Commun. 6 7083 (2015).

38. Gasparini, N. et al. An alternative strategy to adjust the recombination mechanism of organic photovoltaics by implementing ternary compounds. Adv. Energy Mater. 5, 1501527-1571534 (2015).

39. Kirchartz, T. \& Nelson, J. Meaning of reaction orders in polymer:fullerene solar cells. Phys. Rev. B 86, 165201 (2012).

40. Cheng, P. et al. Molecular lock: a versatile key to enhance efficiency and stability of organic solar cells. Adv. Mater. 28, 5822-5829 (2016).

41. Cheng, P. et al. Alloy acceptor: superior alternative to PCBM toward efficient and stable organic solar cells. Adv. Mater. 28, 8021-8028 (2016).

42. $\mathrm{Li}, \mathrm{N}$. et al. Abnormal strong burn-in degradation of highly efficient polymer solar cells caused by spinodal donor-acceptor demixing. Nat. Commun. 8, 14541 (2017).

43. Pearson, A. J. et al. Critical light instability in CB/DIO processed PBDTTTEFT:PC ${ }_{71}$ BM organic photovoltaic devices. Org. Electron. 30, 225-236 (2016).

44. Tremolet de Villers, B. J. et al. Removal of residual diiodooctane improves photostability of high-performance organic solar cell polymers. Chem. Mater. 28, 876-884 (2016).

45. Yan, Y. et al. Light-soaking-free inverted polymer solar cells with an efficiency of $10.5 \%$ by compositional and surface modifications to a low-temperatureprocessed $\mathrm{TiO}_{2}$ electron-transport layer. Adv. Mater. 29, 1604044-1604052 (2017).

46. Zhang, X., Li, W., Yao, J. \& Zhan, C. High-efficiency nonfullerene polymer solar cell enabling by integration of film-morphology optimization, donor selection, and interfacial engineering. ACS Appl. Mater. Interfaces $\mathbf{8}$, 15415-15421 (2016).

47. Cha et al. An efficient, "burn in" free organic solar cell employing a nonfullerene electron acceptor. Adv. Mater. 29, 1701156-1701160 (2017).
48. Gasparini, N. et al. Burn-in free nonfullerene-based organic solar cells. $A d v$. Energy Mater. 7, 1700770-1700777 (2017).

49. Collins, B. A. et al. Molecular miscibility of polymer-fullerene blends. J. Phys. Chem. Lett. 1, 3160-3166 (2010).

50. Zhang, J. et al. Conjugated polymer-small molecule alloy leads to high efficient ternary organic solar cells. J. Am. Chem. Soc. 137, 8176-8183 (2015).

\section{Acknowledgements}

D.B. thanks the Helmholtz Association and Julich Forschungszentrum for financial support via Helmholtz Postdoctoral Fellowship. T.K. acknowledges continuous support from Uwe Rau. A.W. and I.M. thanks EC FP7 Project SC2 (610115), EC FP7 Project ArtESun (604397), and EPSRC Projects EP/G037515/1, EP/M005143/1, T.K. acknowledges support from the DFG (grant KI-1571/2-1).

\section{Author contributions}

D.B. fabricated, characterized solar cell devices, and prepared the manuscript. N.G. performed TPV, CE, and stability measurements and helped with manuscript preparation. C.H.T. carried out XRD, PL, and TAS experiments. M.N. carried out TEM measurements. N.W. performed dynamic SIMS measurements. X.S. did the light intensity and EQE measurements. A.W. and W.Z. synthesized the small-molecule acceptors and helped with manuscript preparation. All authors discussed the results and commented on the manuscript. J.R.D. supervised TAS measurements. T.K., C.J.B., and I.M. revised the manuscript.

\section{Additional information}

Supplementary Information accompanies this paper at https://doi.org/10.1038/s41467018-04502-3.

Competing interests: The authors declare no competing interests.

Reprints and permission information is available online at http://npg.nature.com/ reprintsandpermissions/

Publisher's note: Springer Nature remains neutral with regard to jurisdictional claims in published maps and institutional affiliations.

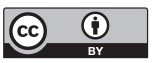

Open Access This article is licensed under a Creative Commons Attribution 4.0 International License, which permits use, sharing, adaptation, distribution and reproduction in any medium or format, as long as you give appropriate credit to the original author(s) and the source, provide a link to the Creative Commons license, and indicate if changes were made. The images or other third party material in this article are included in the article's Creative Commons license, unless indicated otherwise in a credit line to the material. If material is not included in the article's Creative Commons license and your intended use is not permitted by statutory regulation or exceeds the permitted use, you will need to obtain permission directly from the copyright holder. To view a copy of this license, visit http://creativecommons.org/ licenses/by/4.0\%

(C) The Author(s) 2018 